\title{
Signifikant bessere lokale Kontrolle
}

Hintergrund und Fragestellung: In dieser Studie wurde untersucht, ob eine adjuvante Bestrahlung der Lymphknoten (LK) bei Melanompatienten die lokale Kontrolle signifikant verbessert.

Patienten und Methode: Randomisiert wurden 250 Patienten mit erhöhtem Risiko für ein Lymphknotenrezidiv nach vorheriger Lymphadenektomie, welches wie folgt definiert wurde: Anzahl der befallenen Lymphknoten: $\geq 1$ Parotis-

\section{Originalie}

Burmeister BH et al. Adjuvant radiotherapy versus observation alone for patients at risk of lymph-node field relapse after therapeutic lymphadenectomy for melanoma: a randomised trial. Lancet Oncol. 2012;13(6):589-97.
$\mathrm{LK}, \geq 2$ zervikale LK, $\geq 3$ inguinale LK; Kapseldurchbruch; maximale LK-Größe $\geq 3 \mathrm{~cm}$ für zervikale bzw. $\geq$ $4 \mathrm{~cm}$ für axilläre und inguinale LK. Die adjuvante Radiotherapie (RT) des befallenen Lymphabflussgebiets erfolgte zwölf Wochen nach
Lymphadenektomie, mit einer Gesamtherddosis von $48 \mathrm{~Gy}$ in 20 Fraktionen (vier Wochen).

Ergebnisse: Studienkonform behandelt wurden 217 Patienten (109 mit adjuvanter RT vs. 108 im Beobachtungsarm). Bei einem medianen Follow-up von 40 Monaten reduzierte sich die lokale Lymphknotenrezidivrate durch die adjuvante Strahlentherapie signifikant: 20/109 Rezidive im RT-Arm versus 34/109 Rezidive im Beobachtungsarm ( $\mathrm{p}=0,041)$. Bezüglich des progressionsfreien und Gesamtüberlebens zeigte sich kein Unterschied.

Schlussfolgerung der Autoren: Die adjuvante Radiotherapie nach Lymphadenektomie verbessert bei Patienten mit erhöhtem Lokalrezidivrisiko die Kontrollrate signifikant und sollte solchen Patienten immer angeboten werden.

Schlüsselwörter: Melanom - adjuvante Radiotherapie - Lymphadenektomie

\section{- Kommentar von Gregor Goldner, Wien, Österreich}

\section{„Bei hohem Rezidivrisiko adjuvante Strahlentherapie empfehlen“}

Der Stellenwert einer adjuvanten Bestrahlung bei Melanompatienten war bislang umstritten. Bis zuletzt stand eine einzige randomisierte Studie aus dem Jahr 1978 zur Verfügung, die jedoch keinen Vorteil hinsichtlich lokaler Kontrolle oder Überleben zeigte [1]. Weitere Auswertungen zur adjuvanten Strahlentherapie nach Lymphknotendissektion bei Melanompatienten waren zumeist retrospektiv bzw. aus einzelnen Kliniken, und deren Ergebnisse kontrovers [2, 3].

Basierend auf den Resultaten einer vorangegangenen Phase-II-Studie zur adjuvanten Radiotherapie nach Lymphadenektomie bei Melanompatienten (TROG 96.06; [4]) wurde die aktuell publizierte Studie initiiert. Erstmalig konnte nun mit einem randomisierten Vergleich klar nachgewiesen werden, dass durch eine zusätzliche Bestrahlung zur Lymphadenektomie das Risiko eines LK-Rezidivs nach drei Jahren signifikant reduziert wird, hier nämlich von 31 auf 19\%. In der multivariaten Analyse zeigte sich die Ausdehnung des Kapseldurchbruchs als signifikanter Risikofaktor für die lokale Kontrolle (14\% Rezidive bei RT ohne Kapseldurchbruch vs. 32\% Rezidive bei RT mit ausgedehntem Kapseldurchbruch).

Obgleich trotz verbesserter lokaler Kontrolle das progressionsfreie Überleben als auch das Gesamtüberleben nicht verbessert wurden, sind doch die weiteren aktuellen Auswertungen der Studie von Bedeutung. Die Akutnebenwirkungsrate (Grad 3/4) war im RT-Arm mit 19\% Dermatitis bzw. $2 \%$ Schmerzen moderat. Es ist zu erwarten, dass sich in der Folge durch die optimierte lokale Kontrolle auch die Lebensqualität verbessern wird, nämlich durch Vermeidung von Lymphödem, Ulzera und Bewegungseinschränkung. Doch die Auswertung der Spättoxizität und Lebensqualität stehen noch aus. Im weiteren Krankheitsverlauf wird sich auch zeigen, inwieweit sich durch die Etablierung einer effektiven Systemtherapie diese durch den Einsatz der adjuvanten Radiotherapie verbesserte lokale Kontrolle auch in einer signifikanten Verlängerung des progressionsfreien bzw. Gesamtüberlebens niederschlägt. Aktuell sollte, basierend auf den nun vorliegenden Daten der ramdomisierten TROG-Studie, allen Melanompatienten mit einem hohen lokoregionalen Rezidivrisiko eine adjuvante Strahlentherapie nach Lymphadenektomie angeboten werden.

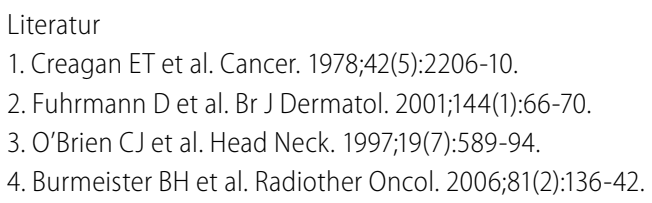

Erstpubliziert in Strahlenther Onkol. 2013;189(1):95-6.

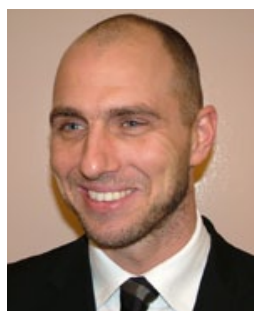

PD Dr. Gregor Goldner

Klinik für Radioonkologie, Medizinische Universität Wien Wien, Österreich Gregor.Goldner@akhwien.at 